
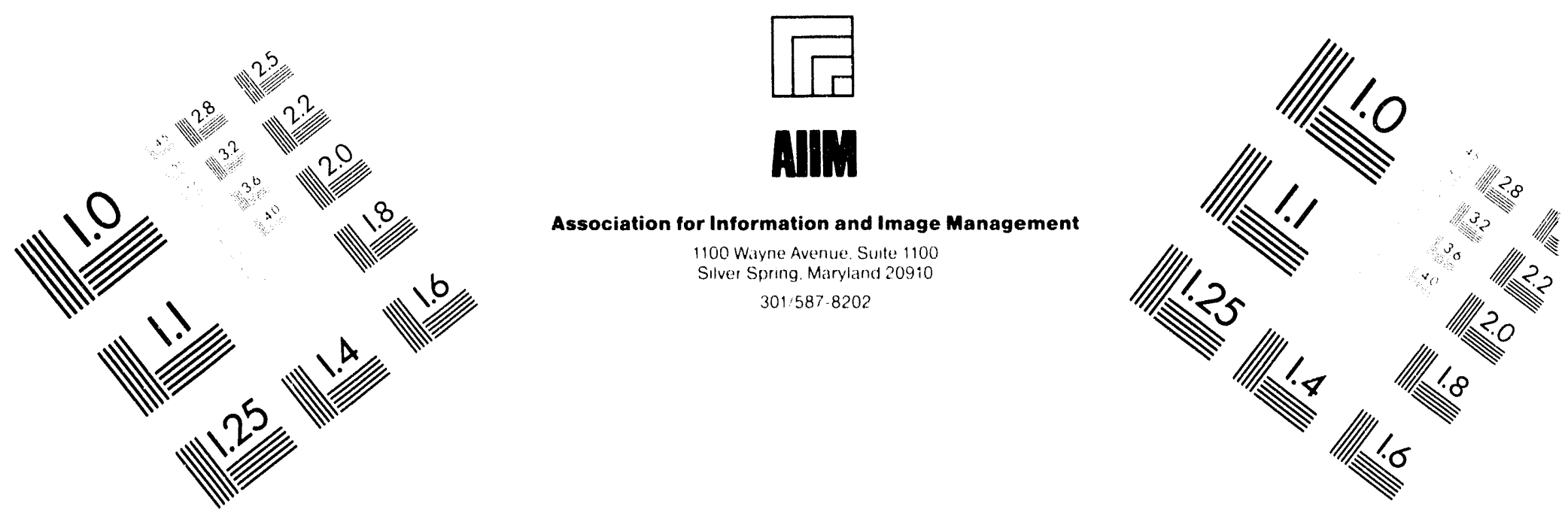

\title{
Centimeter
}

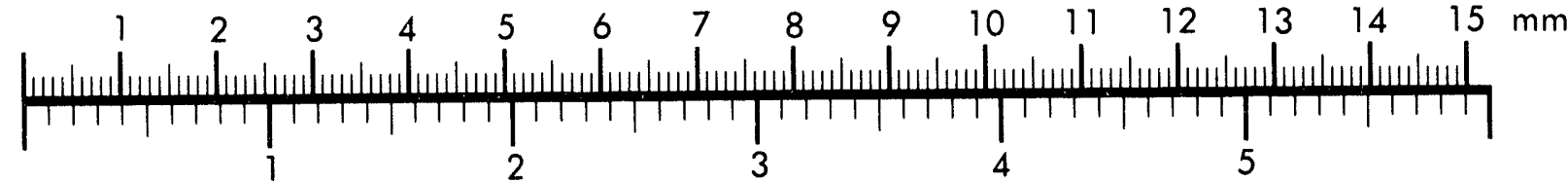

Inches


MANUFACTURED TO AIIM STANDARDS BY APPLIED IMAGE, INC.

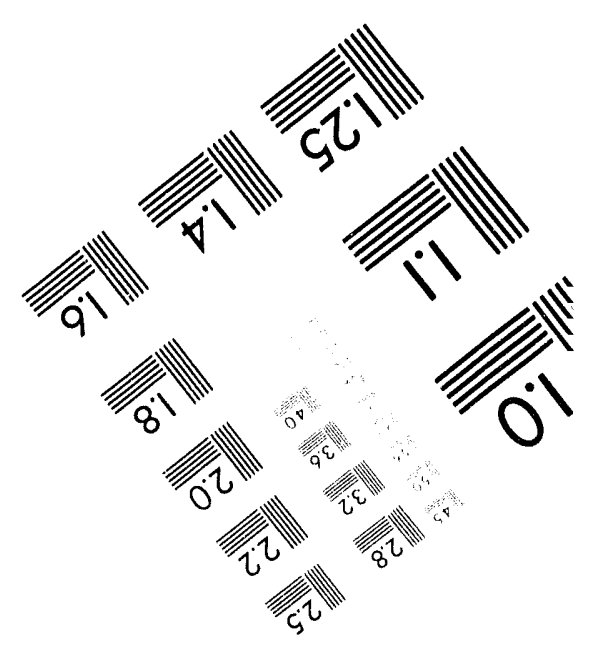






\section{REVIEWS OF COMPUTING TECHNOLOGY: SOFTWARE OVERVIEW (U)}

by

W. R. Hartshorn

Savannah River Site

Aiken, South Carolina 29808

A. L. Johnson

This paper was prepared in connection with work done under the above contract number with the U. S. Department of Energy. By acceptance of this paper, the publisher and/or recipient acknowledges the U. S. Government's right to retain a nonexclusive, royalty-free license in and to any copyright covering this paper, along with the right to reproduce and to authorize others to reproduce all or part of the copyrighted paper. 


\section{DISCLAIMER}

This report was prepared as an account of work sponsored by an ageacy of the United States Governmeat. Neither the United States Governmeat nor any agency thereof, nor any of their employees, makes any warranty, express or implied, or assumes any legal liability or responsibility for the accuracy, completeness, or usefulness of any information, apparatus, product, or process disclosed, or represents that its use would not infringe privately owned rights. Refereace hereia to any specific commercial product, process, or service by trade name, trademark. manufacturer, or otherwise does not necessarily constitute or imply its endorsemeat, recommendation, or favoriag by the United States Government or any agency thereof. The views, and opinions of authors expressed berein do not necessarily state or reflect those of the United States Government or any agency ihereof.

This report has been reproduced directly from the best available copy.

Available to DOE and DOE contractors from the Office of Scientific and Technical Information. P. O. Box 62. Oak Ridge. TN 37831: prices available from (615) $576-8401$.

Available to the public from the National Techaical Information Service, U. S. Deparmeat of Commerce, 5285 Por Royal Rd. Spriagfield. VA 22161 


\section{Reviews of Computing Technology:}

\section{Storage Overview (U)}

by W. R. Hartshorn

WSRC-IM-90-83-14

January 5, 1994

Authorized Derivative Classifier



G. F. Hayhoe

Westinghouse Savannah River Company

P. O. Box 616

Aiken, SC 29802 


\section{Table of Contents}

1 Introduction $\ldots \ldots \ldots \ldots \ldots \ldots \ldots \ldots \ldots$

1.1 Abstract ..................... 1

1.2 Summary ....................... 1

1.3 Scope ......................... 3

2 Current Storage Practices, Environments, Policies, and Issues ........................ 5

2.1 Central Computer Facility $(\mathrm{CCF}) \ldots \ldots \ldots$

2.1.1 IBM MVS (Unclassified or Open) . . . . . . . . 7

2.1 .2 Cray ...................... 10

2.1 .3 IBM 4381 CFS $\ldots \ldots \ldots \ldots$

2.1.4 VAX Systems (Office Support Systems) . . . . . . . . 13

2.1 .5 Tape Library . . . . . . . . . . . . . . . . . . . . 14

2.2 Departmental Systems . . . . . . . . . . . . . . . 17

2.3 Workstations/Servers/Desktop Systems . . . . . . . . . . . 18

2.4 Backup Analysis . . . . . . . . . . . . . . . 20

3 Migration Strategy $\ldots \ldots . . . \ldots . . \ldots 21$

3.1 Near-term Situation and Impacts . . . . . . . . . . . . . 22

3.1 .1 All Platforms . . . . . . . . . . . . . . . 22

3.1.2 Central Computer Facility . . . . . . . . . . . . . . 24 
3.1.3 Departmental Systems $\ldots \ldots \ldots \ldots \ldots$

3.1 .4 Servers/Workstations $\ldots \ldots \ldots \ldots \ldots \ldots$

3.2 Strategy . . . . . . . . . . . . . . . 27

3.2.1 All Platforms and Environments . . . . . . . . . 28

3.2.2 Central Computer Facility . . . . . . . . . . . 28

3.2.3 Departmental Systems _ . . . . . . . . . . . . . 30

3.2.4 Workstations/Servers/Desktops . . . . . . . . . . 30

4 Industry and Technology Trends . . . . . . . . . . 31

4.1 The Storage Hierarchy $\ldots \ldots \ldots \ldots$

4.2 Redundant Array of Inexpensive Disks (RAID) . . . . . . . 33

4.3 Optical . . . . . . . . . . . . . . . 35

4.4 Tape $\ldots \ldots \ldots \ldots \ldots \ldots \ldots \ldots \ldots \ldots$



4.6 Migration . . . . . . . . . . . . . . . 39

5 Future Environment $\ldots \ldots \ldots \ldots \ldots \ldots \ldots \ldots \ldots$

5.1 Overview . . . . . . . . . . . . . . . 41

5.2 Networked Environment . . . . . . . . . . . . . . 42

5.2.1 Integrated Platforms on Highspeed Network $\ldots \ldots .42$

5.2.2 Automatic Data Movement . . . . . . . . . . . 42

5.3 Data Policies . . . . . . . . . . . . . . . . . . 42

5.3.1 Data Administration Effects on Storage . . . . . . . 42

5.3.2 Data Administration Effects on Networks _. . . . . 42

5.3.3 Naming Conventions . . . . . . . . . . . . . . . . 43

5.3.4 File Attributes Specified at Creation . . . . . . . . 43

5.3.5 Frequency of Update . . . . . . . . . . . . . . . . 43 
6 Glossary $\ldots \ldots \ldots \ldots \ldots \ldots \ldots \ldots \ldots \ldots \ldots \ldots \ldots$

7 References $\ldots \ldots \ldots \ldots \ldots \ldots \ldots \ldots \ldots \ldots \ldots$ 
This page left intentionally blank. 


\section{Introduction}

\subsection{Abstract}

The Savannah River Site Computing Architecture states that the site computing environment will be standards-based, data-driven, and workstation-oriented. Larger server systems deliver needed information to users in a client-server relationship. Goals of the Architecture include utilizing computing resources effectively, maintaining a high level of data integrity, developing a robust infrastructure, and storing data in such a way as to promote accessibility and usability. This document describes the current storage environment at Savannah River Site (SRS) and presents some of the problems that will be faced and strategies that are planned over the next few years.

\subsection{Summary}

The current total computer disk storage at SRS on all computing platforms is over 1.5 terabytes, based on an estimate using the completed computer inventory. A conservative estimate of the investment represented by this amount of storage is $\$ 15$ million.

The following are the key conclusions of this study:

Delay storage acquisitions as long as possible.

- Maximize utilization of current available storage.

- Automate storage management processes.

- Delay purchase; buy only what is needed.

- Emphasize automation software and hardware processes.

- Utilize less manpower-intensive computer operations. 
- Employ tape silos.

- Procure new storage architecture devices (for example, hot pluggable).

- Employ compression where applicable.

- Continue WISDOM implementation.

- Provide improved storage backup mechanisms.

- Encourage automatic tape stackers and tape silos.

- Migrate data to network/server and backup to attached storage.

Storage, like other computer resources, must be upgraded regularly, as part of the equipment life cycle. Large expenditures and potential. savings can be anticipated if a strategy is developed to maximize the utilization and devote the storage resources to the proper computing platform. Projections in this document indicate that the site will invest over $\$ 5$ million in disk storage over the next four fiscal years (FY-94 through FY-97).

This discussion involves all computing platforms in use at SRS except for the process control and digital control systems. These systems are dedicated to the processes that they control and therefore do not necessarily follow the guidelines and strategies proposed in this document. 


\subsection{Scope}

The current distribution of disk storage at the SRS as shown in Figure 1 indicates that over 55\% of the total storage capacity is in PCs and workstations.



WORKSTATIONS $3 \%$

Figure 1: Storage analysis by computing platform

- PCs include a wide variety of hardware including Apple (various Macintosh models), IBM (various PC and PS/2 models), and others (Compaq, HP, DEC), and total over 14,000 devices.

- Workstations include SUN Sparcstations, VAXstations, small VAX computers, RS/6000, Silicon Graphics, and Intergraph, and total approximately 288 devices.

- Midrange systems include medium, large, and very large VAX systems supporting ALL-IN-1; medium, large, and very large VAX systems supporting departments; and Intergraph computers. These total almost 200 systems. 
Mainframe systems include several IBM computers (currently 3090, 3081, and 4381) and a Cray X-MP.

The investment in computer hardware is over $\$ 115$ million, including network and computing support. This figure does not include the investment in computer facilities (buildings, computer rooms, etc.) or computer support equipment (cooling, power, expendables, etc.). This total also does not include the process control and digital control systems installed in production areas on the site.

This study covers the requirements for storage, backup, and archiving of data.

The following definitions will clarify some special terminology used in this document.

\section{Backup}

The process of creating a copy of a file which may reside locally or at a server. Backups protect the user from loss because of hardware or software failure and accidental deletion. Backup differs from archiving because backup may be automatic and archiving is always user-directed. Backup may be done in a variety of techniques, the most popular being full and incremental. Logs constitute a form of backup for some database applications. While the full backup takes longer to perform, the incremental, which processes only files which have changed sir se the last operation, requires longer for recovery since multiple incremental versions must be applied before recovery is complete.

\section{Recovery}

The process of restoring a copy of the user's data from the backup inventory. Recovery may be used to replace an existing file or to create a new file with a new name.

\section{Archiving}

A user-directed function that provides long-term storage for data unlikely to be referenced in the near future. A copy of the file is made, and the original file may be deleted. This action may appear to be automatic when files move according to the parameters set by the system storage administration group.

\section{Retrieval}

A user-directed function that returns to the user an explicitly requested version of a file that had been archived, and optionally deletes it from the archive. Again, this action may appear to be automatic when files move according to parameters set by the system storage administration group.

Definitions of other terms can be found in Chapter 6 . 
Figure 2 indicates the rapidly expanding growth that has taken place in Central Computing Facility storage requirements in the last few years.

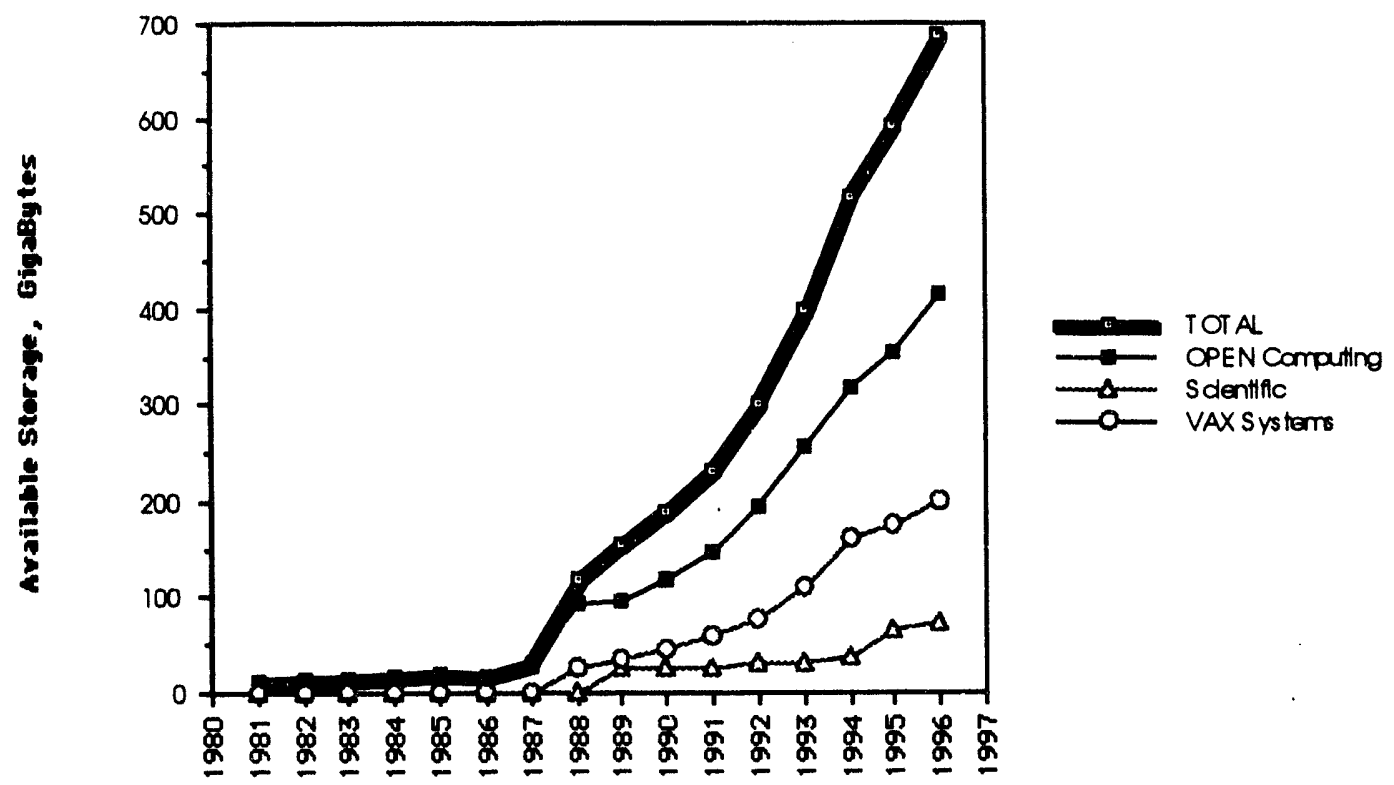

Figure 2: Central Computing Facility storage systems

Storage growth was suppressed in the 1985-87 time frame because there was no space to house additional equipment and construction of additional space could not be authorized. When the new computer facility at $703-44 \mathrm{~A}$ was completed, storage expansion took off and is projected to continue at about the same pace as new online applications and databases are developed. 


\subsubsection{IBM MVS (Unclassified or Open)}

The primary handler of the unclassified workload for the Central Computer Facility is an IBM 3090-400J computer system with 264 GB of storage attached. Over 7,000 users of this system generate a workload with the characteristics (typical month) shown in the following table.



The primary applications running under the CICS transactional processing system are:

- IBARS-Integrated Budget, Accounting, and Reporting System tracks cost budgets and expenditures links directly to PCS for cost detail. (Typical day shift activity is 40 concurrent sessions.)

- CLS-The Consolidated Labor System tracks manpower and activity codes. (Typical day shift activity is 70 concurrent sessions.)

- PCS-The Procurement Cycle System tracks requisitions, order processing, receiving, and payment for goods and services. (Typical day shift activity is 135 concurrent sessions.)

- WMS-The Work Management System tracks manpower and equipment maintenance data and schedules. (Typical day shift activity is 70 concurrent sessions.)

- Other systems-Tesseract (Payroll), System W, and INTELLECT, and the Development, and Acceptance environments. (Typical day shift activity is 150 concurrent sessions for all these.) 
CCF storage devices require controllers; the typical configuration is shown in Figure 3.

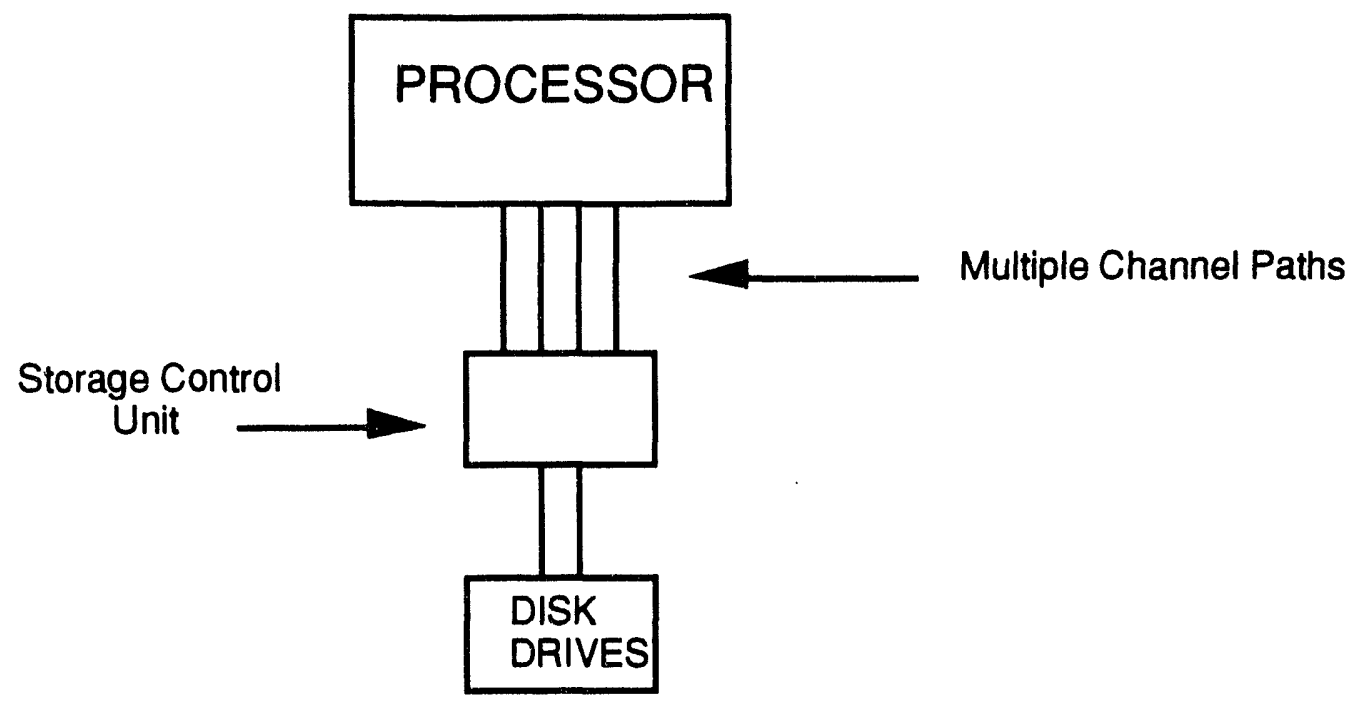

Figure 3: Typical storage device configuration

A total of 58 storage control units are used in the current configuration which connects 124 IBM 3380 disk drives of various models and 40 IBM 3390 disk drives.

Allocation of this DASD is:

- system and system maintenance: $20 \%$

- database: $\mathbf{5 5 \%}$

- user and production: $17 \%$

- scientific, backup, and reserve: $8 \%$

The availability of some online systems is reduced to read-only access to allow backup to take place between 2:00 am and 5:00 am daily. Other applications are disabled so that good backups are maintained. This practice permits full backup of databases and incremental backups of other data.

Daily backups ( 6 days/week) move about $39 \mathrm{~GB}$ of data to approximately 200 tapes. More complete backups of the entire DASD environment each weekend move 80 to $90 \mathrm{~GB}$ of data to approximately 500 tapes.

Other policies are used to control data space. Files which have not been accessed for a period of time (for example, 60 days) are migrated to tape. 
Users may request that archived files be returned to disk. This operation is usually performed overnight. Users are typically allowed a little under $3 \mathrm{MB}$. The unclassified system has about 7,100 authorized users-over half of whom require access only to the productions systems (IBARS, PCS, WMS, CLS, etc.) and therefore do not require any online space since their work involves entry or query of records in databases already allocated in support of those systems. This results in the relatively low amount (under $10 \mathrm{~GB}$ ) of user data space.

Periodic projections are requested so that capacity planning can be attempted. Availability of capital funds is another consideration in this scenario, in that space may not currently be deliverable because no funds were authorized for procurement of equipment. This process of planning for computer equipment and funding is part of the Information Technology Resources Long Range Plan development and approval process. This process has not alwayc been adequate in the past because approved plans do not always result in approved funding. This problem can lead to capacity shortfalls in both processing power and storage.

The following issues impact the MVS unclassified mainframe.

\section{Equipment obsolescence}

The storage control units and most of the devices attached to the unclassified system are not capable of supporting the new asynchronous commands that are required to separate the devices from the processing units using fiber optic channel paths that can be over $15 \mathrm{~km}$ long.

\section{- Backup problem}

The time to make the necessary backups of database and system volumes requires that the applications be inactive or made read-only so that the databases are static during the backup period. The time required grows as databases get larger. To combat this problem, faster and higher capacity tape devices will help, as will using incremental dumps.

\section{- Disaster recovery problem}

Because of tape formats, compression, etc., it is essential to have matching format tape units at a recovery site so that backup data can be restored.

\section{- Systems Managed Storage}

A plan to install and implement Systems Managed Storage has been developed. SMS is currently in operation and will be phased in to do more and more of the dataset allocation and control. The software will continue to be upgraded with 
new releases that are more stable and have additional features.

\section{- Impact of partitions on storage}

New mainframes can be partitioned into separate logical processors. These logical partitions impact storage in that a separate physical storage device is required for each logical system. In addition, the new storage devices seem to be ever increasing in their capacity per volume. This means that certain volumes (for example, the load or system residence volume) which are usually replicated in various versions on any individual system now require more space (most of which is wasted) just to provide alternate systems. Careful planning and alternate modes of operation, including automating building of residence volumes, may be a viable alternative.

\subsubsection{Cray}

The very high performance requirements of the Cray systems environment have dictated a storage environment different from other systems in use at SRS. The Cray has 19.5 GB of local high-speed disk storage. The operating system and standard user applications use approximately 3 GB. Some of the remaining space is allocated to the users, and the rest is generally available temporary space. All user disk space on the Cray is regaided as temporary or unreliable in that it is not backed up and the system managers reserve the right to reconfigure at any time. Long-term, backed-up storage is the user's responsibility. The IBM 4381 CFS provides this service.

The disk configuration of the X-MP/EA 2/64 consists of 16 DD-49 drives each of which has a $1.2 \mathrm{~GB}$ capacity for a total of $19.5 \mathrm{~GB}$. The disks attach through DCU-5 interfaces, each of which can support up to four drives.

\subsubsection{IBM 4381 CFS}

The IBM 4381 Common File System (CFS) provides file services for the Cray and Savannah River Technology Center (SRTC) VAX cluster systems. It contains 13 GB of storage and four IBM 3480 tape drives. The primary features of CFS are that disk space appears unlimited to the user and files may reside on either disk or tape, transparent to the user. Files are written to tape or disk as needed.

The CFS system operates the DataTree software that was originally developed for Los Alamos by General Atomics. Version 56 was the original version installed at SRS in 1989. After the initial installation, DISCOS took over sup- 
port of this product but has now turned support over to OpenVisions Technology. The last version (version 60) was installed in 1992. No future upgrades of this system are contemplated.

Figure 4 indicates the steady growth that has taken place since the Central File System was implemented.

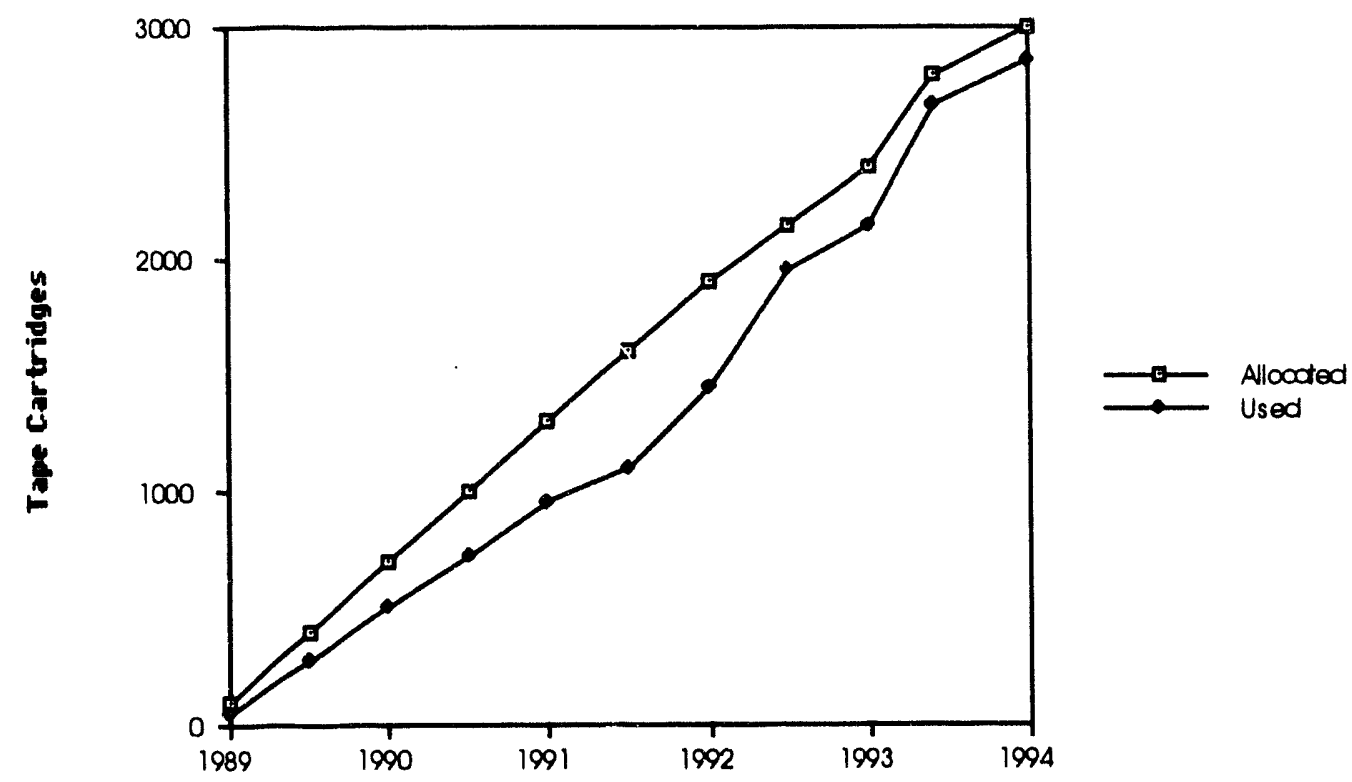

Figure 4: Central File System tape archive

The following problems and issues affect this platform:

- The user interface to DataTree is difficult. It is a line-oriented interface with poor online help facilities. The user must remember a large number of commands.

- Even though there are fewer than 50 regular users of this system, the expectation is for continual growth in the number of tapes as files are archived to tape storage.

- Other needs are a modest growth in disks so that the system can handle larger files online and an expanded number of tape units so that more tapes can be handled concurrently.

- The current CFS system is limited. Possible future systems are discussed below.

- UNITREE is an improved version of file management for UNIX systems now marketed by OpenVisions Technology, the supplier of DataTree. 
UNITREE is a UNIX-based hierarchical file and storage management system for networked, multivendor computing environments. Within the UNITREE system, user files are automatically migrated between magnetic disk, cartridge robot, and archival tape, allowing users to select the best mixture of high performance and low cost storage for the application. Since the software keeps track of storage files, users need not know whether a requested file resides on disk or other media.

UNITREE features an object-oriented, client-server configuration which supports parallel $\mathrm{V} / \mathrm{O}$, multiple file servers, and separate data and control paths. UNITREE also offers storage security and access control features. The UNITREE software provides storage service to large network environments including systems of workstations, minicomputers, and supercomputers. The system relies on industrystandard network hardware and protocols, providing Network File System and File Transfer Protocol service across FDDI, Ethernet, HYPERchannel, HIPPI, and Ultranet networks.

Features of UNITREE include the following:

- unlimited file size, unlimited data storage capacity, unlimited number of files

- multi-level file caching

- automatic file migration

- direct data path to shared peripherals

- globally unique file naming

- shadowing of directory information

- file transaction journaling

- peripheral replacement with minimal interruption

- client software packages

- Distributed File Transfer Protocol

- UNITREE Client Disk Manager 


\section{- UNITREE Shared Peripheral Manager}

- AFS, formerly the Andrew File System developed at Carnegie Mellon University, has been developed as a commercial product by Transarc, Inc. This product uses Kerberos authentication, offers caching of files at the record level to reduce network load, and can replicate volumes or entire servers. OSF has chosen AFS as its distributed file system technology, implying that all the major manufacturers (DEC, IBM, and HP) are committed to supplying it. The downside is that AFS is a new system with a smaller user community and a considerable number of startup problems.

- DFS, Distributed File System, is the future file system that will conform to the OSF criteria for distributed file system technology. It essentially builds and expands on the AFS features, and with the full support of major vendors will probably be the system of choice in a few years.

\subsubsection{VAX Systems (Office Support Systems)}

The primary use of the office support systems is to provide 15,000 information workers with access to electronic messaging and other services through the ALL-IN-1 system.

The office support systems were started as a result of a study conducted in 1985 to determine site information needs. DEC's ALL-IN-1 software and VAX/VMS hardware were selected as the SRS office support platform in 1986 through a competitive procurement process. The original systems were designed with the assumptions that the user population would approach a maximum of 6,000 , electronic mail messaging would be the primary application, and load growth would be optimally distributed between clusters in three areas of the site.

The information needs now provided cover a variety of services far beyond the original design expectations in addition to electronic messaging. These include access to services such as Compuserve, Dialog, BRS, the site telephone directory and lists of assisting personnel, file transfer, and word processing capabilities.

Office support services are provided by Digital Equipment Corporation mainframes. The larger mainframes operate in clusters to increase reliability. If one should fail, then operation can continue in a degraded mode of operation. Upgrade of these systems to provide ALL-IN-1 and Videotext support for all users who have access to the SRS network is continuing. Restrictions in capi- 
tal equipment funding have delayed some upgrades. Currently, the network is supported by seven major nodes consisting of 14 computers with $60 \mathrm{~GB}$ of storage attached.

The BASIS file and text retrieval system, which includes access to site records, incidents, plant drawings, and library databases; DOE databases; and others via keyword searches, is supported on other office support computer systems with $25 \mathrm{~GB}$ of storage.

Backups are performed nightly, and shadowed disk techniques are used to reduce the significance of a potential disk failure. Four incremental backups are performed during the evening on weekdays, while a full backup is performed each weekend.

The following issues affect this platform:

Additional processing capacity is required tc support the additional concurrent user workload. ALL-IN-1 is used as the standard mechanism for rapid dissemination of information to employees throughout the site.

- Additional storage capacity is needed to support additional user workload.

- On several occasions, users have sent messages containing classified information through ALL-IN-1. This requires suspension of operation until the system can be sanitized of this information. Procedures need to be developed to reduce the time to recover from these outages.

- New office support and mail systems that are networkbased rather than host-based are now being piloted as the office systems of the future. These products permit improved routing of documents, forms, audio, and video, in addition to messaging. Migration to these new office systems will require careful planning.

\subsubsection{Tape Librany}

The tape library has existed since the first computing systems were established at SRS.

Tape usage has changed over the years from essentially $100 \%$ tape jobs, since no direct access storage existed, to today's situation where users and systems have tapes assigned but efforts are being made to eliminate tape usage in favor of direct access disk storage.

Reel tapes have undergone transitions over time as the standard tape formats have shifted from low density 7 -track recording to higher density and higher track formats. 
The latest transition has been the conversion from 3420 reels $(2,400$ foot, 9 track, 6,250 bpi density) to 3480 cartridges which hold about 550 feet of tape. Both the 3420 and 3480 formats will handle a nominal $200 \mathrm{MB}$ of data per reel or cartridge.

Whercas the total number of tapes was slowly growing (there were fewer than 8,000 tapes in 1987), the advent of the Central File System and the conversion to cartridges pushed the library volume to nearly 26,000 in 1991 . After dropping somewhat as the number of older 3420 reels were converted and not replaced, the total number of tapes is again increasing. The number of 3420 reels is expected to level at under 1,000 reels.

The distribution of the current iape inventory is:

\begin{tabular}{|c|c|}
\hline \multicolumn{2}{|c|}{ Current Tape inventory } \\
\hline Unclassifled 3420 tape reels & 2,000 \\
\hline Unclassifled 3840 tape reels & \\
\hline 3081 & 1.100 \\
\hline VAX & 1.800 \\
\hline 3090 & 14,400 \\
\hline 4381 & 920 \\
\hline DataTreө & 2,800 \\
\hline New & 800 \\
\hline User tapes & 330 \\
\hline
\end{tabular}


Figure 5 illustrates the trends in tape volumes on various computing platforms during the past seven years.

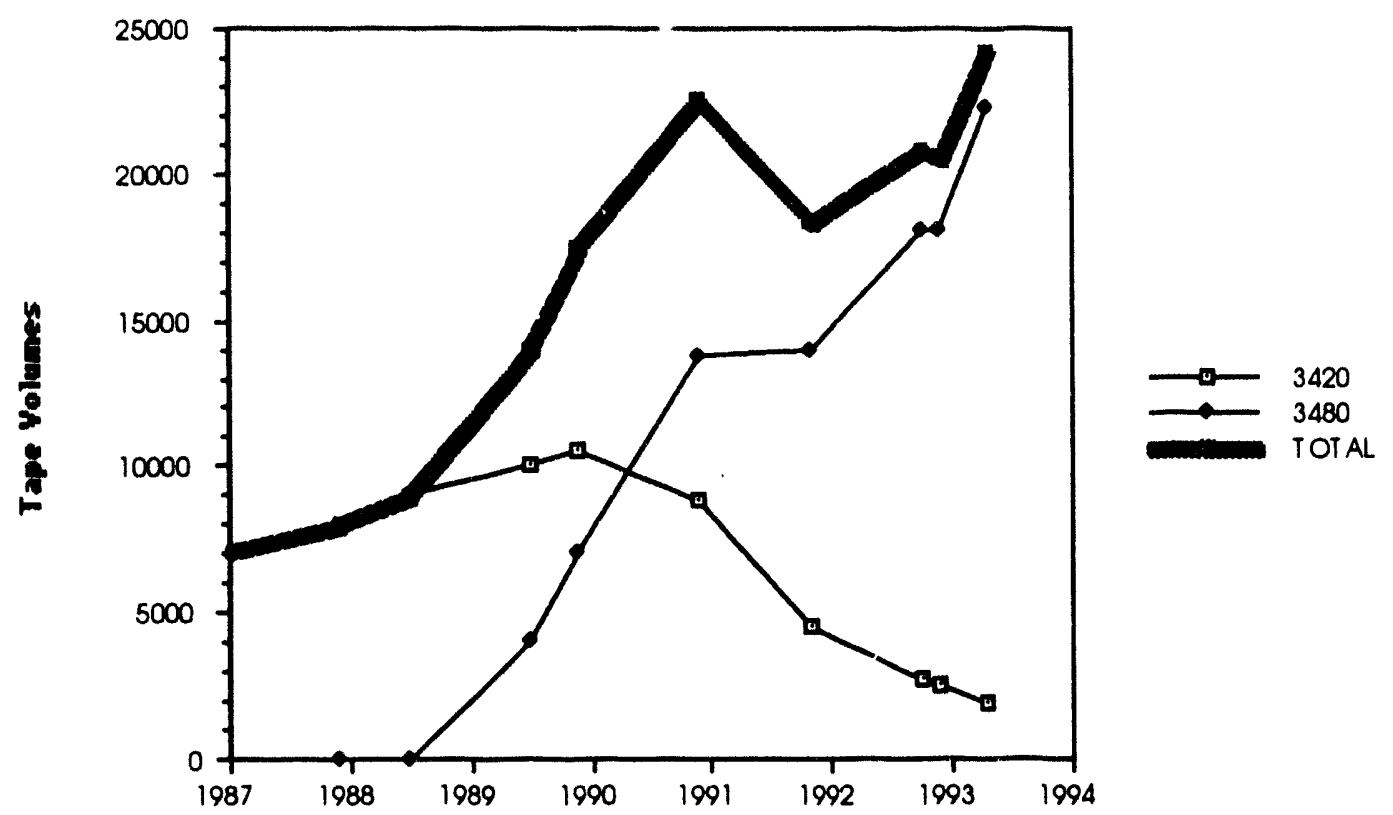

Figure 5: Central Computing Facillty tape library

The cost of 3420 reels is (was) about $\$ 12$ per 2400 reel. The cost of 3480 cartridges is about $\$ 12$ per cartridge. The investment in media is therefore $\$ 366,000$.

Currently the tape library occupies 1,500 square feet of space. Space for the library which includes environment and security controls standard for computer facilities runs about $\$ 200$ per square foot. This results in a tape library space value of $\$ 300,000$. Management of the library costs an additional $\$ 100,000$ per year.

Mounting a tape, based on industry studies and input into the IRM Chargeback System is $\$ 2.20$ per mount.

Tape mounts for disk dumps and data backup account for $84 \%$ of all tape data activity in the unclassified CCF. There are about 1,700 tape mounts per week on the 3090 alone. 


\begin{tabular}{|l|l|}
\hline \multicolumn{2}{|c|}{ Tape Library Cost/Value Summary } \\
\hline Investment Costs & \\
\hline Media & $\$ 366,000$ \\
\hline Space & $\$ 300,000$ \\
\hline Operating Costs & \\
\hline Management & $\$ 100,000 /$ vear \\
\hline Mounts & $\$ 180,000 /$ year \\
\hline
\end{tabular}

\subsection{Departmental Systems}

Over 200 computer systems at SRS qualify as departmental systems. For the most part these systems were procured and established in the 1980s and are primarily VAX systems from Digital Equipment Corporation.

A survey sent to the system managers of these systems revealed that $75 \%$ of the systems had a database associated with the primary application on the system. Database systems broke down as follows:

- Ingres: 35 licenses

- Oracle: 96 licenses

- RDB: 54 licenses

Oracle is the choice for the site standard in this tier of computing. Special purpose systems will be allowed for process control or other specific site systems.

Databases averaged occupying only about $7 \%$ of the total available disk capacity on these midrange systems; however, one system was running a database that used $90 \%$ of its disk capacity.

Most of the departmental systems are in good shape storage-wise, with an average of $33 \%$ available disk not currently being utilized and available for growth and expansion. Only $16 \%$ of the system managers anticipated any need to expand their disk capacity over the next two years, but indicated that 
if budgets were constrained during that period, they could probably live with their current capacity.

All of the systems have data backups performed on a routine basis. The typical backup, using $8 \mathrm{~mm}$ tape units with a capacity of 2 GB per cassette, is performed weekly (though some systems have daily/nightly backups), and takes about four hours. The backup is usually automated.

Most systems have a policy of rotating through a series of tapes, and some have rather elaborate policies for moving tapes to different locations so that a backup is available in the event of a disaster.

The following issues impact departmental systems:

Management of the backup media is not as sophisticated as that used by the CCF. The system manager keeps a list of the tapes, or the information (date, etc.) is written on tape containers or label.

- A better tape management system that will encompass these tapes is desirable to let system managers participate in this management technique over the SRSnet network.

\subsection{Workstations/Servers/Deskłop Systems}

The extensive use of personal computers and workstations has been a fairly recent phenomenon. Improvements in speed, reliability, and operating system functionality have led to the development of mission-critical applications on this tier. The demands of these applications have, in turn, forced the workstation toward higher performance, increased memory, and larger local storage requirements.

The individual nature of the personal computer, however, inhibits cooperative or workgroup applications. This fact has led to substantial development in the Local Area Network (LAN) use of file and print servers. These servers are less capable than the office support system VAX machines listed above, but because of their localized and specialized nature provide a highly cost-effective solution to workgroup computing and are efficient in the use of the network.

A typical territorial or zone file server might be used to provide file services for about 100 to 150 personal computer workstations. It would likely contain a read-only space for applications and full read-write space for both individual and shared data.

It is important to note that this file space is accessible from various workstation types (IBM DOS-based machines, Macintosh computers, and UNIX workstations). This feature provides a degree of file-interchange transparency and machine interoperability, limited mostly by the application software. 
There are several choices for partitioning the file system between the workstations and the servers. Each of these partitions represents a different tradeoff between system cost, the number of workstations per server, and the ease of managing the user files.

- A swapful workstation allocates the virtual memory swap space and temporary files on its local disk. The operating system's files and user files remain on the server. This approach increases the network traffic to the server, leaving the issues of system management relatively uncomplicated. For example, in this configuration, the local disk does not have to be backed up. However, execution of an operating system command still requires access to the remote server.

- A dataless workstation adds the operating system's files to the workstation's local disk. This configuration reduces the workstation's demand on the server, thus making it possible for a single server and network to support more workstations. While it is still not necessary to back up the local disk, the system is more difficult to administer. For example, system updates must be distributed to all of the workstations.

- A diskfull workstation places all but some frequently shared files on the workstation. This choice yields the lowest demands on the server but causes the biggest problems for system management. The personal files on the local disk need to be backed up, requiring either local intervention by the user or significant network traffic during backup operations. In addition, this design limits the ability of the user to move from one workstation to another.

An alternative approach leverages the lower cost semiconductor memory to make feasible large file caches ( $8 \mathrm{MB}$ or more) in the workstation. These caches provide an effective way to circumvent network latencies if the network protocols allow file writes to be decoupled from communications with the server. The approach, called diskless workstations, has been demonstrated in some networks which report a five- to ten-fold increase in workstations per server over more conventional client/server organizations.

The design currently being implemented by the Information Resource Management Department (IRM) at SRS calls for a dataless workstation which stores only the system (operating system, GUI, networking software) and temporary (virtual memory swap, print cache, application cache) files on the local storage. The local file server provides a source of (updated current version) application software and a location for personal and group data storage. This space is centrally managed and backed up, providing a safe location for mission-critical data.

Network data management will assume more IRM resources over time. IRM will be asked to decrease not increase headcount. Therefore, automation is essential. The system must, as much as possible, manage itself. 


\subsection{Backup Analysis}

\begin{tabular}{|c|c|c|}
\hline \multicolumn{3}{|c|}{ Backups Currently Performed at SRS } \\
\hline Environment & Ampunt of & $\begin{array}{l}\text { Nymber of } \\
\text { por Week }\end{array}$ \\
\hline CCF & & \\
\hline 3090 & $297 \mathrm{~GB}$ & 1.700 \\
\hline 3081 & $82 \mathrm{~GB}$ & 400 \\
\hline 4381 & $25 \mathrm{~GB}$ & 125 \\
\hline Cray & $3.5 \mathrm{~GB}$ & 20 \\
\hline CFS & $3 G B$ & 55 \\
\hline Office systems & $80 \mathrm{~GB}$ & 460 \\
\hline Departmental & $100 \mathrm{~GB}$ & 100 \\
\hline Workstations, etc. & $250 \mathrm{~GB}$ & 250 \\
\hline Total & $840 \mathrm{~GB}$ & 3,110 \\
\hline
\end{tabular}




\section{Migration Strategy}

This section discusses some of the future SRS projections and developments that may impact some of these forecasts. Capabilities that will have major impact on the storage requirements if they are implemented are being contemplated.

This section has two parts. The first discusses situations and impacts projected to occur on each of the computing platforms over the next few years. The storage projections are covered here. The second part discusses the cost impact of these computing storage plans and the areas where effort needs to be directed in each computing environment to implement these plans. 


\subsection{Near-term Situation and Impacts}

\subsubsection{All Platforms}

Hardware costs are steadily decreasing, as Figure 6 illustrates.



Figure 6: Storage costs

Technology cycles for computer products are decreasing (The time from product introduction to the point where its residual value hits $50 \%$ is typically under two years, compared with periods twice that time five years ago).

Impacts on Storage: More data will be added to the corporate inventory. Today, only an estimated $10 \%$ of all enterprise data is coded for on-line retrieval. With each passing day, more of the enterprise data is being placed in machine-readable form, primarily due to the growth of end-user computing. A greater percentage of documents will end up in electronic format and even "filed" documents from record storage will be scanned into computer storage to facilitate information searches and retrieval. Associated with the distrib- 
uted data is a cost of locating the data desired. The need for centralized management of a diverse set of decentralized processing solutions is obvious if cost is an issue.

Model office scenarios with voice and video, for improved communications and training, will add to the storage demands even with the imposition of severe compression algorithms.

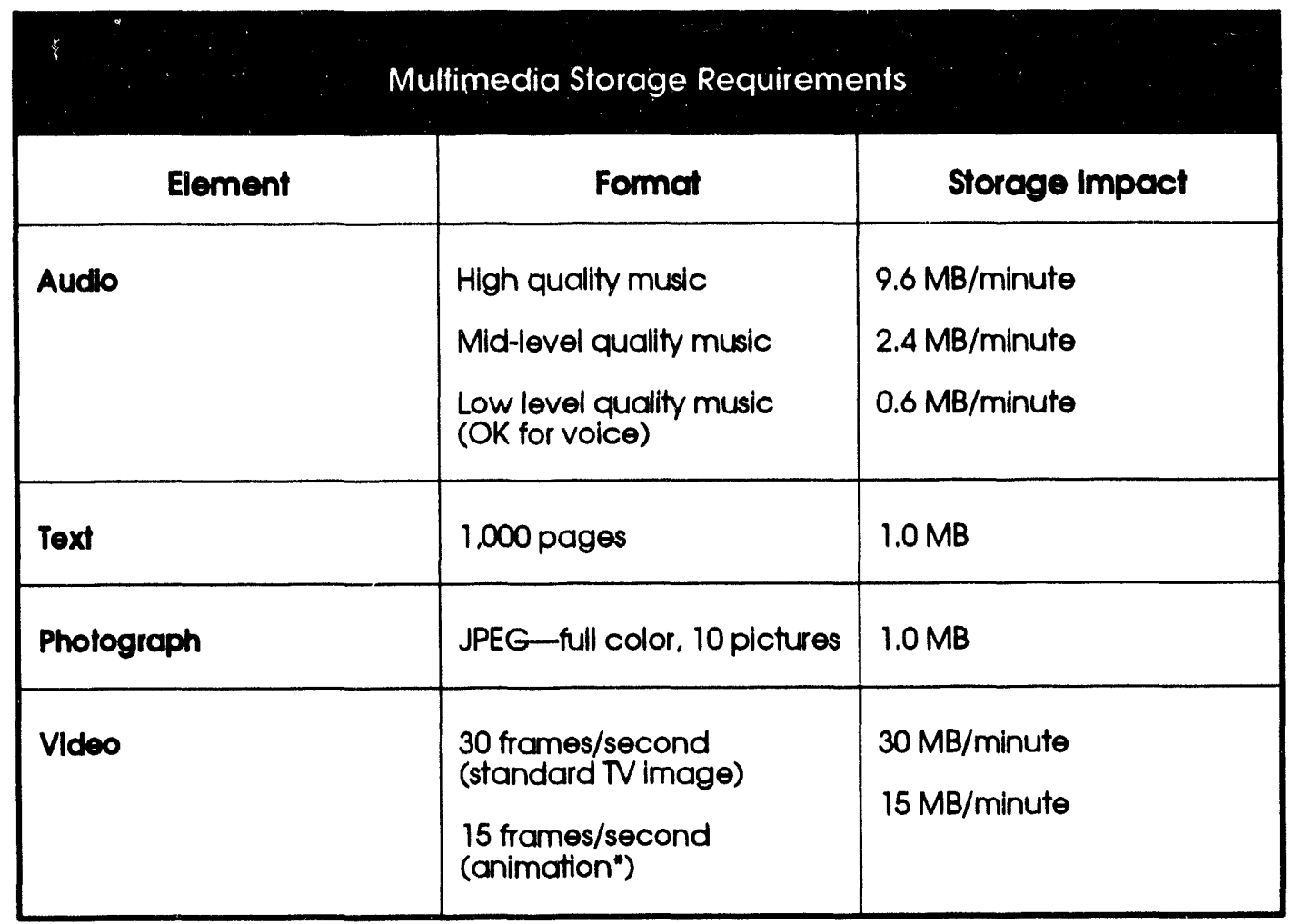

* Reduced image size and compression can reduce storage impact.

Replication-Mail files, some containing image formats, would be replicated across network post offices. The impact to a simple network equates to about a seven-fold increase in storage needs; a large network may have 100 servers, so a $3 \mathrm{MB}$ file might require $300 \mathrm{MB}$ of storage depending on the sophistication of the mail or distribution system.

Information Warehouse-The warehouse concept that is now in pilot stage will extract data from the primary repositories and create data structures more closely aligned with the functional or subject area needed for management queries and to give more responsive access. The estimated storage impact can be up to a $50 \%$ increase in space depending on how deeply this concept penetrates into the site application systems. This concept will also impact on the file archive system in that these new data structure files will be archived, probably on a monthly basis, to facilitate access to this information.

The overall growth in computer systems storage projected for the Savannah River Site is charted in Figure 7. 




Figure 7: SRS storage projection

Most of the storage will continue to exist on the desktop, not because it is needed or is highly utilized there, but its cost is so low that site standard workstations will have 300 megabytes of storage because that is the industry standard. Growth will occur in all the platforms except those currently categorized as departmental. These systems will merge into the network (WISDOM) area as workgroup servers as the current host platforms become obsolete.

\subsubsection{Central Computer Facility}

While ADP budgets remain fairly flat, the hardware component is shrinking. Storage is taking a larger percentage of the hardware budget. The Gartner Group predicts that the storage component will grow from $45 \%$ of the total hardware expenditures in 1992 to $60 \%$ in 1996 . While the industry average compound annual growth rate of mainframe storage is dropping below the traditional 30 percent, the rate still requires doubling disk storage capacity every 30 to 36 months. Unchecked, storage managers can expect to have as much unused capacity in the glass house in 30 months as they have today because of waste for over-allocation, interspersed space, inefficient block sizes, and volume reserves. At the same time, storage capacity on the network is growing faster than that on the mainframe. Trends indicate that the growth rate in number of physical data files is exceeding the capacity growth, but 
storage management personnel cannot grow because of budget constraints and lack of skilled people. Also processor performance improvements continue to exceed storage performance improvements.

Planning and disaster preparation will take on increased importance. Both functions will benefit from automation, but the percent of resource consumption will increase. Backup, space management, and performance management will consume fewer resources as automation increases. Acquisition efforts for network storage will become increasingly important.

A key to handling the storage is using the latest software tools that automate the management process according to installation-defined policies, thus reducing the staff required to handle/manage data files as the quantity grows with storage demands. Implementing Systems Managed Storage is the first step in providing this capability. When these products are running comfortably, the next step is to implement the hierarchical storage manager, which will monitor data set usage and archive inactive data to nearline or offline storage. Further integration of function will result from the application of the remote media manager, which will keep track of the tape volumes and can also track tape volumes from other systems.

The ALL-IN-1 user population has now peaked and will level off. As SRS personnel rosters are reduced and SRS missions are redirected, the ALL-IN-1 support role will be to provide improved service and information access to the remaining user population. Projections are that this will be accomplished by adding major support nodes for processing support and additional storage.

\subsubsection{Departmental Systems}

Most of these systems are currently connected to the site network. Alternative backup schemes should be tried to determine the network impact of moving data to other systems or servers periodically.

Reorganization initiatives prompted by the Contractor Business Systems Review analysis and DOE directives will encourage more standardized and consolidated approaches to providing computer applications to answer departmental needs.

\subsubsection{Servers/Workstations}

File server storage space will become increasingly important as users migrate data away from the workstation. Data compression will not provide enough increase to balance this demand. Approximately $10 \mathrm{MB} / \mathrm{user}$ will be provided for general user space and $10 \mathrm{MB}$ /user will be managed workgroup space. File servers will typically be configured with 1 to $2 \mathrm{~GB}$ of disk space. 
The networked workstation and server concept is being implemented across SRS. Server backup will be increasingly important since user files of critical site data exist only on the server. Effective management and control of this backup (and recovery) process will be essential as the number of servers increases.

Because the basic design calls for dataless workstations, the major emphasis on workstation storage should be to provide system and swap space. Current OS requirements would indicate that 40 to $60 \mathrm{MB}$ per workstation is adequate. Future OS needs would push these numbers to 80 to $120 \mathrm{MB}$. However the workstation will still be configured with 200 to $300 \mathrm{MB}$. Perhaps some of this space can be utilized as cache to buffer the network traffic and response. 


\subsection{Strategy}

The following table tracks the actual and projected growth in storage devices in the SRS computing environments. The capacity requirements are then multiplied by the storage cost to arrive at the projected expenditures for the next few yoars.

\section{Storage Projections}

\begin{tabular}{|c|c|c|c|c|c|c|c|c|}
\hline Year & & & FY-92 & FY-83 & FY-84 & FY.86 & FY.88 & FY.97 \\
\hline \multicolumn{9}{|l|}{ Business } \\
\hline Computing & GB & & 230 & 300 & 390 & 470 & 580 & 650 \\
\hline \multirow[t]{2}{*}{$\$ / M B$} & & & 11 & 9 & 6 & $\mathbf{3}$ & 2.5 & 2 \\
\hline & & & & $\$ 630,000$ & $\$ 540,000$ & $\$ 240,000$ & $\$ 275,000$ & $\$ 140,000$ \\
\hline \multicolumn{9}{|l|}{ Departmental } \\
\hline \multirow[t]{2}{*}{ Computing } & GB & & 316 & 375 & 400 & 300 & 250 & 200 \\
\hline & & & & $\$ 424,800$ & $\$ 120,000$ & & & \\
\hline Network & Worksta & GB & 39 & 50 & 80 & 110 & 140 & 200 \\
\hline Computing & Servers & GB & 25 & 100 & 200 & 250 & 275 & $\mathbf{8 0 0}$ \\
\hline (\& Desktop) & PC's & GB & 735 & 900 & 1500 & 1800 & 2000 & 2400 \\
\hline \multirow[t]{2}{*}{$\$ / M B$} & & & 3 & 2.5 & 1.5 & 1 & 0.6 & 0.5 \\
\hline & & & & $\$ 627,500$ & $\$ 1,095,000$ & $\$ \$ 80,000$ & $\$ 153,000$ & $\$ 242,500$ \\
\hline \multicolumn{9}{|l|}{ Office } \\
\hline Computing & GB & & 130 & 250 & 300 & 325 & 350 & 350 \\
\hline \multirow[t]{2}{*}{ ALL-IN-1 } & GB & & 75 & 110 & 160 & & & \\
\hline & & & & $\$ 697,500$ & $\$ 300,000$ & $\$ 37, ; 00$ & $\$ 31,250$ & $\$ 0$ \\
\hline \multicolumn{9}{|l|}{ Scientific } \\
\hline \multirow{2}{*}{ Computing } & GB & & 32 & 40 & 60 & 100 & 150 & 200 \\
\hline & & & & $\$ 72,000$ & $\$ 120,000$ & $\$ 120,000$ & $\$ 125,000$ & $\$ 100,000$ \\
\hline TOTAL (GB) & & & 1,507 & 2,016 & 2,890 & 3,365 & 3,746 & 4,300 \\
\hline
\end{tabular}


The estimated expenditures to procure this amount of storage is:

FY.93: $\$ 2,451,800$

FY-94: $\$ 2,540,000$

FY-95: $\$ 1,166,250$

FY-96: $\$ 772,500$

FY-97: $\$ 736,500$

\subsubsection{All Platforms and Environments}

SRS must work toward systems which will integrate the operation of computers and use the strengths of each system to its best advantage. IRM will begin to manage of backup and archiving of files from a multitude of platforms, including departmental systems. Automation and user-friendly system interfaces will be required in this environment.

Management controls or whatever methodology works will reduce file space by purging old files, etc. Files will be migrated from disk to tape or floppies, etc.

SRS must also automate controls or plan for implementation of software managed controls to reduce manpower intensive operations.

Any acquisitions should be delayed as long as possible, since prices will steadily drop. The same dollars will buy more storage in future. However, SRS should not over-purchase for the same reason, but buy only what is absolutely necessary to keep the operation going.

SRS must also analyze the cost/benefits of faster and higher capacity tape backup systems. SRS needs to develop alternatives to handling $2,500+$ tapes per week and to implement recovery tests to demonstrate that data can be recovered for the backups.

\subsubsection{Central Computer Facillity}

SRS must install and implement products that will automate the storage management process to a large extent. The first step in this direction is the Systems Managed Storage product. This product operates only in the MVS/ESA operating system environment, so plans need to be made to have platforms in the CCF that operate with ESA. The CCF has already made most of the prerequisite steps to get to SMS-a major step was completed with the transition to RACF which instituted new and revised data set naming conventions for the entire facility. Work is currently in progress to put SMS into full operation by the end of CY-1994. 
IBM has announced additional capabilities for this product with new releases anticipated in 1993 and 1994. These improved products now combine the Systems Managed Storage, the Data Set Services (DSS) functions, the Hierarchical Storage Manager (HSM), and a new Remote Media Manager (RMM) under the umbrella of the single product, DFSMS, as separate functional components. Incorporation of these newer versions of this software should be accomplished as the next improvements in a steady progression of the current plan.

This software will be the base that will handle the storage and backup management functions in a cost-effective manner for both media and manpower. It will also provide the base so that the CCF mainframes could participate in a more global manner to support network and network-attached devices, including departmental computer systems.

SRS should also focus on automation. From the existing automation products in the CCF, procedures can be developed that begin to have a more global view of systems and systems storage. Initial tests with software to handle the movement of files from network servers to the mainframes will be evaluated.

Studies of tape usage and cost/benefit analysis of alternative modes of operation (like tape silos) should be performed to determine the most effective operation for the future. In this analysis, new, denser tape formats, like 3490E or optical tape, should be considered.

Disk devices need to be converted to new technology in a time frame consistent with need. The new technology features include ESCON non synchronous operations attachment, concurrent copy, and cache management. These enhanced functions need to be supported, but in due time. They need to be analyzed in light of decreasing costs for DASD and the shrinking life cycle for these products.

Savannah River Technology Center personnel are currently running AFS software in a limited environment. These pilots are necessary to gain experience in the performance of this file system and to determine the learning curve characteristics associated with it. Understanding the impact of Kerberos authentication techniques which will carry forward into the DFS and DCE environments is also a necessary step.

IBM has announced their intention of supporting AFS and DCE files in the DFDSM product which is to be available in 1993. IBM intends to implement selected elements of OSF-DCE across platforms, including AIX/6000 and AIX/ESA, which is an operating system built upon OSF OSF/1 with additional IBM enhancements. We would hope that, in a few years, MVS/ESA would provide similar support. 


\subsubsection{Departmental Systems}

Departmental systems will tend to migrate to departmental servers as server hardware is installed throughout SRS and most functions and applications can be supported via client/server interfaces. Most departmental systems will not be replaced at the end of their life cycle.

\subsubsection{Workstations/Servers/Desktops}

The existing $14,000+$ devices which constitute this category are rapidly aging. Replacement of older, obsolete devices needs to be continued on a regular basis using standard desktop and server configurations.

Even though storage cost is steadily declining, the need for large amounts of storage is discouraged, since data will be routinely stored (backed up, migrated, etc.) on the workgroup server. Desktop storage will primarily be used for local caching to improve performance of applications and provide temporary storage for multimedia (audio and video) applications since video applications put severe demands on network data transfer capacity. Applications using multimedia and video for training and communications could become common. Therefore, some download capability is needed to provide a "buffer" for consistent user viewing at the terminal. Video compression hardware is expected to be economical by 1995 , and the SRS infrastructure should support the high transfer rates that will make such applications feasible in 1996.

Networking of users and their workstations at SRS has been accepted as part of the computing architecture at SRS. The WISDOM concept of providing this implementation will spread throughout the site over the next few years. The following table shows the projected implementation of WISDOM.

\begin{tabular}{|c|c|c|c|c|c|}
\hline & \multicolumn{4}{|c|}{ Projected Implementation of WISDOM } & \\
\hline & FY92 & FY93 & FY94 & FY95 & FY96 \\
\hline Wispom & 1.000 & 3,800 & 6.900 & 9.00 & 9.600 \\
\hline$\underset{\text { Number of }}{\text { Nonts }}$ & 10 & 40 & 65 & 100 & 125 \\
\hline Stgrgipe & 25 & 100 & 190 & 250 & 275 \\
\hline
\end{tabular}




\section{$4 \quad$ Industry and Technology Trends}

This section describes the hardware and software technologies that will affect storage decisions over the next few years.

\subsection{The Storage Hierarchy}

The storage hierarchy is traditionally modeled as a pyramid (see Figure 8), with a small amount of expensive, fast storage at the pinnacle and larger capacity, lower cost, and lower performance storage as we move towards the base.

In general, there are orders of magnitude of difference in capacity, access time, and cost among the layers of the hierarchy. For example, main memory storage is measured in megabytes, costing approximately $\$ 50$ per megabyte, and can be accessed in small numbers of microseconds. Secondary storage, usually implemented by magnetic disk, is measured in gigabytes, costs less than $\$ 5$ per megabyte, and is accessed in tens of microseconds (milliseconds). The operating system can create the illusion of a large, fast memory by judiciously staging data among the levels. However, the organization of the storage hierarchy must adapt as magnetic and optical recording methods continue to improve and as new storage devices become available.

In the workstation/server environment of today, the storage hierarchy is distributed across multiple devices, with the network playing an integral part in linking them together. Most of the semiconductor memory in the server can be dedicated to the cache function because a server does not host conventional user applications. The file system "metadata," that is, the data structures describing how logical files are mapped onto physical disk blocks, can be held fast in semiconductor memory. This represents much of the active portion of the file system. Thus, disk latency can be avoided while servicing user requests. 


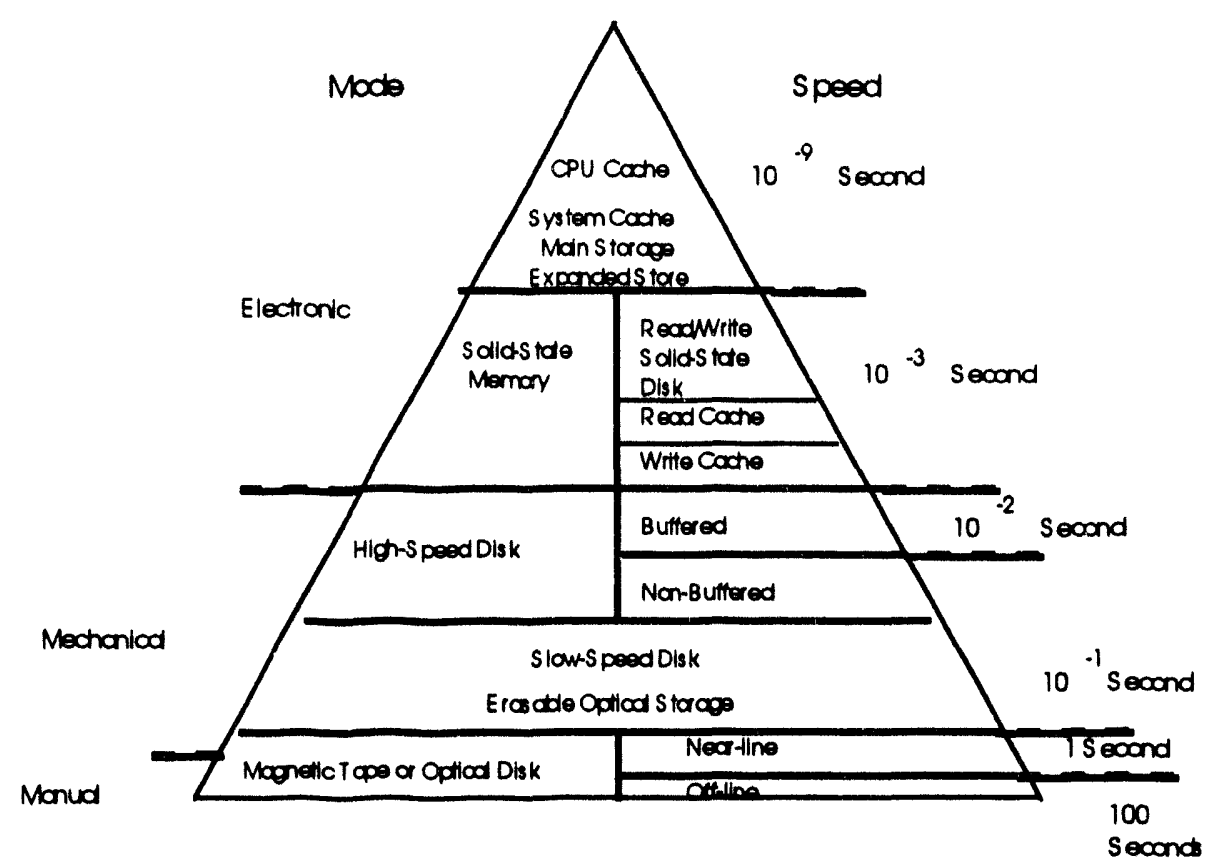

Figure 8: Storage components

The critical challenge for workstation/server environments is the added latency of network communications. Network latency times are comparable to those of magnetic disk, and are measured in small tens of milliseconds. One possible solution places small, high performance disks in the workstation, with larger, potentially slower disks at the server.

If most accesses can be serviced by the local disks, the network latencies can be avoided altogether, improving workstation performance and responsiveness. However, there are several choices for how to partition the file system between workstations and servers. Each of these partitions represents a different tradeoff among system cost, the number of workstations per server, and the ease of managing the workstation's files.

Technological improvements are occurring in many areas affecting storage. The medium for disks is changing from iron oxide which supports a maximum density of about 100 million bits per square inch to chromium cobalt alloys which can store 2 gigabits per square inch. Scientists now predict that by using magnetoresistance technology, disks will be capable of storing up to 10 gigabits per square inch by the end of this decade. Reliability is also improving, with mean time between failure rates of five years being touted as more intelligence is being built into device control units. Controllers now have the 
capability of detecting errors prior to failure and maintaining units during operation so that device failures don't have the impact on continuous operations that they once did.

\subsection{Redundant Array of Inexpensive Disks (RAID)}

Because of the rapidly decreasing form factor of magnetic disks, it is becoming attractive to replace a small number of large disk drives with very many small drives. The resulting secondary storage system can have much higher capacity since small format drives traditionally obtain the highest area densities. And since the performance of both large and small disk drives is limited by mechanical delays, it is no surprise that performance can be dramatically improved if the data to be accessed is spread across many disk actuators or is heavily cached. Disk arrays provide a method of organizing many disk drives to appear logically as a very reliable single drive of high capacity and performance.

RAID uses groups of off-the-shelf, inexpensive disk drives. RAID disk systems provide higher performance than similar capacity conventional disk systems. RAID is defined in levels. Each RAID level is a different way of combining disk drives to achieve an improvement in performance, reliability, or both.

Level 0 - This methodology involves data striping, by scattering individual data across several hard disks. This method can dramatically increase data throughput and can be useful in scientific applications where large amounts of data are transferred. This technique can also reduce the transfer time to more acceptable times.

- Level 1-Disk mirroring takes two disks in place of every one that would normally be used for storage. This provides a continual backup of storage. Recovery time after a disk crash is almost non-existent because there is no need to reconstruct any records. This level allows for hot-swapping without shutting down normal operations.

- Level 2 - This RAID technology incorporates a self-correcting parity coding system developed by Hamming. Hamming Code uses four information bits and three check bits per character. This approach requires a lot of disk space but nut quite as much as disk mirroring. An example of its use might be using a seven disk array with four drives containing striped data and three dedicated to error correction. If a drive fails, however, you pay a penalty in downtime since the drive must be replaced and lost data recreated.

Level 3 - Error correction capability is added to the hardware controller, reducing the need for so many drives dedicated to parity data. In the example of a seven-disk array system, tevel 3 would carry striped data on six. The other 
disk would carry the error-correction codes. This RAID level is good for tasks where large amounts of data are being transferred.

- Level 4-This level protects data by a combination of hardware and single-disk parity error correction, so that it has the same ratio of data to parity disks as in Level 3. User data is striped in parallel across several disks. It is suitable for read-intensive applinations.

- Level 5 - This level is popular because it provides both good performance and good data integrity. This is accomplished by striping both data and parity bits across multiple drives. This is the only RAID level with parallel writes. Level 5 is implemented entirely in software, making it cheaper than the other RAID levels, especially for existing disk systems. User data and parity data are interleaved and striped across several disks.

- Level 6 - This additional level of RAID tolerates simultaneous failure of any two disks in a RAID-5 type array. It provides double error correction with Reed-Solomon Codes and provides support for asynchronous operation.

- Level 7 - This level extends asynchronous operation support. It means that exclusive of and independent of the host, or multiple host paths, the embedded operating system manages all $\mathrm{V} / \mathrm{O}$ transfers asynchronously acruss the RAID 7 data and parity drives.

- Level 8 - This may be a RAID-5 scheme using RAID-3 subunits. 


\subsection{Optical}

Optical storage is a rapidly developing medium, but will probably be used only in special applications only for the next few years, mainly because of the relatively slow data transfer rates and the lack of standards. Currently there are no clear leaders as manufacturers, format standards, or recognized applications.

The primary technologies are:

- write once, read many (WORM)

- ablative

- bubble/blister

- dye-polymer

- phase change

- alloy

- erasable/rewritable

- magneto-optic (MO)

- phase change

- multifunction drives

- hard WORM

- $\operatorname{soft}$ WORM (WO/MO)

- CD-ROM-stamped or etched

- CD-R-WORM in CD format

The primary applications in which optical is finding a niche are in image applications and as replacements for Computer Output Microfilm (COM). One primary advantage of this medium is its anticipated long storage life and fast retrieval relative to filed documents or fiche. Many users are using optical to provide the archival records of its documents and drawing files. These types of decisions need to be made cautiously since real media lifetimes have not yet been established. The problem is, of course, that it takes a long time to 
copy to optical (users are achieving less than 1 GB per hour). Restore rates are very good, however.

Digital optical tape is discussed in the next section.

\subsection{Tape}

Magnetic tape media will be the primary choice for system and data backup through the year 2000 because its low cost, good reliability, and portability will not be challenged during that time period.

The packaging of tape, its recording format, and its transport mechanism is undergoing rapid technological change, just like other areas of computer technology.

The current types of tapes are listed with some of their characteristics.

\section{Current Types of Tapes in Use}

\begin{tabular}{|c|c|c|c|c|c|c|c|c|}
\hline $\begin{array}{l}\text { Medial } \\
\text { Drive }\end{array}$ & Length & Format & Capacity & $\begin{array}{c}\text { Media } \\
\text { Cost }\end{array}$ & $\begin{array}{c}\text { Drive } \\
\text { Cost }\end{array}$ & Cost/GB & $\begin{array}{c}\text { Transfer } \\
\text { Rate }\end{array}$ & $\begin{array}{l}\text { Tape } \\
\text { Time }\end{array}$ \\
\hline 4mm DAT & $60 \mathrm{~m}$ & helical & $1.3 \mathrm{~GB}$ & $\$ 15$ & $\$ 3,000$ & $\$ 11.60$ & $186 \mathrm{~KB} / \mathrm{s}$ & $2 \mathrm{hr}$ \\
\hline $8 \mathrm{~mm}$ cartridge & $112 \mathrm{~m}$ & helical & $2.3 \mathrm{~GB}$ & $\$ 12$ & $\$ 7,000$ & $\$ 5.30$ & $246 \mathrm{~KB} / \mathrm{s}$ & $2.5 \mathrm{hr}$ \\
\hline $1 / 2$ in VHS & $120 \mathrm{~m}$ & helical & $14.5 \mathrm{~GB}$ & $\$ 20$ & $\$ 35,000$ & $\$ 1.80$ & $2-3 \mathrm{MB} / \mathrm{s}$ & $1.6 \mathrm{hr}$ \\
\hline $1 / 2$ in 9 -trk & $2400 \mathrm{ft}$ & 9 track & $200 \mathrm{MB}$ & $\$ 12$ & $\$ 20,000$ & $\$ 60.30$ & $1 \mathrm{MB} / \mathrm{s}$ & $3.3 \mathrm{~min}$ \\
\hline $1 / 2$ in 3480 & $540 \mathrm{ft}$ & 18 track & $200 \mathrm{MB}$ & $\$ 12$ & $\$ 20,000$ & $\$ 60.30$ & $2-3 \mathrm{MB} / \mathrm{s}$ & $1.3 \mathrm{~min}$ \\
\hline $1 / 2$ in $3490 E$ & $1080 \mathrm{ft}$ & 36 track & 2.4 GB & $\$ 15$ & $\$ 50,000$ & $\$ 6.90$ & $9 \mathrm{MB} / \mathrm{s}$ & $4.5 \mathrm{~min}$ \\
\hline 1/2in DLT & $1100 \mathrm{ft}$ & track & $6.0 \mathrm{~GB}$ & $\$ 45$ & $\$ 8,000$ & $\$ 7.60$ & $500 \mathrm{~KB} / \mathrm{s}$ & $3.3 \mathrm{hr}$ \\
\hline 1/4in cartridge & $760 \mathrm{ft}$ & track & $1.3 \mathrm{~GB}$ & $\$ 45$ & $\$ 3,000$ & $\$ 34.60$ & $240 \mathrm{~KB} / \mathrm{s}$ & $1.5 \mathrm{hr}$ \\
\hline D2, R-90 & & helical & 25 GB & $\$ 200$ & $\$ 150,000$ & $\$ 10.00$ & $15 \mathrm{MB} / \mathrm{s}$ & $28 \mathrm{~min}$ \\
\hline
\end{tabular}

A product with good potential is digital optical tape. This write-once technology tape packaged in a 3480 cartridge would have a capacity of 50 GB to 250 GB per cartridge. The form factor of the medium would mean that current tape silos or robots could expand from a capacity of around 1 terabyte to over a petabyte with only the drives requiring replacement. The write-once tech- 
nology, long shelf life, capacity, and high performance make it ideal for storage of large data files and imagery, and for the secure backup of databases.

\subsection{Compression}

Data compression is available in both hardware and software implementations to reduce the storage requirements and/or the transmission time for moving the data from point to point. The problem is that many implementations are proprietary, and no standard is imminent except in video.

Two major methods are used for data compression: statistical (e.g., Huffman coding) and dictionary (e.g., Lempel-Ziv-Welch). Other compression techniques are also discussed below.

Text files can be compressed the most; for example, the text you are reading can be compressed from 50 to $70 \%$, depending on the method used. Dense machine language files can be compressed about a third. Some graphics files leave little room for compacting.

The following are the major compression methods:

- Huffman coding-Statistical compression method that converts characters into variable length bit strings. Most-frequently-occurring characters are converted to the shortest bit strings; least frequent, the longest. Compression requires two passes. The first pass analyzes a block of data and creates a tree model based on its contents. The second pass compresses the data via the model. Decompression decodes the variable length strings via the tree.

- LZW-Widely used dictionary compression method that stems from two techniques introduced by Jacob Ziv and Abraham Lempel in 1977 and 1978. LZ77 scans a fixed length block of data and creates pointers back to data when it repeats. 1778 scans the data and creates a dictionary of repeating phrases. Pointers are created to these phrases.

- JPEG (Joint Photographic Experts Group)_ISO/CCITT standard for compressing a still picture that is based on the discrete cosine transform (DCT) method. It uses variable compression (from 10 to 100 times) based on the resolution quality desired and can achieve $90 \%$ reduction with minimal loss. For example, using a PC with a 1991-vintage JPEG board, a full-color $25 \mathrm{MB}$ image can be compressed into $1 \mathrm{MB}$ in one second.

MPEG (Moving Pictures Experts Group)-ISO/CCITT standard for compressing full motion video. The MPEG image scheme offers more compression than the JPEG scheme, which is largely for still images, because it takes advantage of the fact that full motion video ( 20 to 30 frames/second) is made up of many successive frames consisting of large areas that are not changed-like 
blue sky background. While JPEG compresses each still frame in a video sequence as much as possible, MPEG also performs "differencing," noting differences between consecutive frames. If two consecutive frames are identical, the second can be stored with the appropriate information. MPEG condenses moving images about 3 times more tightly than JPEG.

Some of the more popular compression programs are:

- PKZIP-A software program for compressing files. A database can be reduced by as much as $90 \%$ and a word processing file by about $30 \%$ by using PKZIP.

- TAR-UNIX utility for tape archiving, used in conjunction with compress.

- ZOO-Freeware compression program, including source code, used in UNIX and DOS environments

The following are the major hardware implementations:

IBM IDRC-A hardware compression feature available for 3480 and 3490 tape drives.

- Storage Tech ICEBERG-Inventors of this RAID device claim it stores up to $400 \mathrm{~GB}$ of data (in compressed format) on disks that actually contain $160 \mathrm{~GB}$ of space.

Some have speculated that IBM may come out with a compression technique for channel connected devices, much like the IDRC used for magnetic tape. However this is not likely while IBM is in the disk business, since to introduce compression would essentially eliminate two to three years of DASD acquisitions. It is more likely that some compression aspects will be introduced to enhance performance of IBM's database product, DB2.

Upcoming network operating systems software will provide automated file compression. Compression is typically done at the server prior to the disk write. Decompression might be done at the file server or at the workstation. In the latter case, the compressed file is carried across the network, resulting in reduced network traffic because the file is smaller. The compression algorithm used is intensive in the compressing process rather than in the decompressing process. Because of the file server cache process, there should be little impact on the performance as viewed by the user. The local workstation should be more than capable of performing the necessary decompression.

New additions to the workstation (both IBM and Macintosh platforms) operating system provide for automated, user-transparent data compression. The long term effect might be to reduce the need for larger local storage in the future.

Several vendors offer products to compress packets of data on the network. These are in addition to the products which strip repetitive characters (e.g., blanks) from network data streams. 


\subsection{Migration}

Migration of data to increasingly slower media is one solution to storage costs but is balanced by the costs of management. There is an increasing interest in providing automatic functions to perform data migration based on predetermined rules.

File server and mainframe technology now exists to allow user data to migrate from fast magnetic media to slower magnetic media and then to optical disk or tape based on the amount of time since last access. Although all the data appears to be in the same space, it is actually split across various media, and is recalled to the faster medium when required by the user. 
This page left intentionally blank. 


\section{Future Environment}

This section provides an overview of the future SRS computing environment from the storage perspective.

\subsection{Overview}

The SRS Computing Architecture states that Site computing will be standards based. The file storage environment will increasingly encompass remote storage devices. Remote devices will be accessible via a suite of standard protocols and services built on top of the TCP/IP protocols. Standards affecting storage management include the Open Systems Foundation Distributed Computing Environment (OSF DCE) suite of protocols. OSF DCE protocols complement the traditional suite of TCP/IP protocols. The most important, from the storage perspective, is the Distributed File System (DFS). DFS is based on the Andrew File System. DFS relies on authentication services provided by the security portion of DCE. Security is based on the Kerberos authentication system developed at MIT. Also critical to the function of DFS is the naming service that uses X.500 as the global name service.

Standards for physical storage devices are important as well. Interface standards for disk drives have resulted in the very high-performance, high-reliability RAID configurations. Tape standards have resulted in a multitude of automatic tape handlers for $8 \mathrm{~mm}$ and IBM 3480 tape form factors.

Site computing will be data driven. Thus, the correct data must be available to every user who requires it. It is important that all data be maintained reliably and be accessible with adequate performance.

Site computing will be workstation-oriented. The user interface for all services should be the native interface of the workstation wherever possible. For storage, this implies that remote files and disks be accessed through an interface that is like the native interface for that workstation (i.e., Macintosh systems see AppleShare volumes and PCs see drive letters). 


\subsection{Networked Environment}

\subsubsection{Integrated Platforms on Highspeed Network}

One of the more important features of the contract for the SRS Replacement Telephone System is the installation of robust fiber to provide high speed (greater than $100 \mathrm{MB} /$ second) interarea voice/data communications. Fiber technology is required to provide the speeds for networked responsiveness over moderate distances. Where fiber is not available, high speed digital connections will be utilized to provide a responsive network.

\subsubsection{Automatic Data Movement}

Migration of files and backup will occur based on parameters and specifications set by the network administrator. Rules and policies will be established to migrate data through at least part of the storage hierarchy. Based on usage and data criticality parameters established for the site, data will be migrated from repository to repository as defined and as required.

\subsection{Data Policies}

\subsubsection{Data Administration Effects on Storage}

Data administration policies will affect the capacity required at each computing platform. The following is a partial list of some of the policies that affect the storage capacity:
frequency of backup
- full or incremental backup
- compression of files
- responsiveness required when accessing data
- freeboard capacity requirement

\subsubsection{Data Administration Effects on Networks}

Data administration policies will affect the network capacity (band width) and the network responsiveness, These policies will consider all possible sce- 
narios so that data administration traffic does not overburden the network capacity and result in an unresponsive system from the user perspective.

\subsubsection{Naming Conventions}

File or data set naming conventions will be established so that the user will be coaxed into providing new file names of the correct type and syntax. Versions of the "same" file will be accommodated so that users can readily establish the version they are working with. Since critical data will, by specification, have backup copies generated automatically, the identification of the original version is essential.

\subsubsection{File Attributes Specified at Creation}

When a user creates a file, the attributes which it will have through its lifetime will be established. This process will be as painless as possible, with many attributes being defaults for the typical user. However, the attributes can be queried and changed as necessary. The attributes determine such things as how long the file will be retained, how critical it is, how responsive it should be, etc.

\subsubsection{Frequency of Update}

Certain files will be refreshed periodically from another location. This operation may be triggered by a timer or a particular data change in the master file. 
LSP This page left intentionally blank. 


\section{Glossary}

ACL

Automatic Cartridge Loader.

ACS

Automatic Cartridge System.

\section{Archiving}

A user-directed function that provides long-term storage for data unlikely to be referenced in the near future. A copy of the file is made, and the original file may be deleted.

ATL

Automated Tape Library.

\section{Backup}

The process of creating a copy of a file which may reside locally or at a server. Backups protect the user from loss because of hardware or software failure and accidental deletion.

Backup differs from archiving because backup may be automatic and archiving is always user-directed.

\section{Bpi}

Bits per inch.

Byte

A group of eight bits handled as a logical unit.

CD-ROM

Compact Disk-Read-only Memory. CD-ROM refers to read-only optical disk devices.

Client

A client receives service from a provider. Thus, a client might be a workstation, a LAN server, or even a supercomputer. 


\section{Common Storage Manager}

An outboard, micro-programmable processor that is expected to manage the storage subsystems (e.g., DASD, tape, optical libraries) by the mid1990s. The Common Storage Manager is expected to be the 3990 replacement and to run elements of DFP, as well as functions such as data compression/compacting, logging, and journaling.

\section{CSF}

Common Storage Format. Anticipated method for future systems to store data on $\mathrm{I} O \mathrm{O}$ devices in multiples of $4 \mathrm{~KB}$.

DASD .

Direct-Access Storage Device. DASD is a general term, but typically refers to a magnetic disk device.

DAT

Digital Audio Tape - an emerging technology offering high density.

DCE (Distributed Computing Environment)

A comprehensive integrated set of services that support development, use, and maintenance of distributed client/server applications on a network.

\section{DFP (Data Facility Product)}

DFP is the MVS operating system component that contains the access methods and handles catalog management. This component contains about half of the 10 million lines of code in the operating system and is the property of IBM's Storage Systems Products Division.

DME (Distributed Management Environment)

A set of integrated programs that provides coherent management of systems and networks.

\section{Disk Array}

See RAID.

\section{Dual Copy}

A capability whereby a single host system's write operation causes two mirrored copies of the data to be written to separate devices. Also called Shadow Write (by DEC).

\section{Exabyte}

1,000 terabytes or one thousand trillion bytes.

GB or Gbyte

Gigabyte, 1,000 million bytes or 1 billion bytes.

Hyperchannel

Network Systems Corporation's proprietary high-speed connection between hosts from a wide variety of manufacturers. 


\section{IDRC (Improved Data Recording Capability)}

A tape feature announced by IBM in 1989 that improves the effective capacity for the 3480 and 3490 cartridges.

\section{LPAR (Logical PARtition)}

The PR/SM (Processor Resource/System Management) feature allows the CPU to operate as multiple logical processors, each capable of running a different operating system and applications set.

\section{LU 6.2 (Logical Unit Type 6.2)}

An SNA protocol that is device independent and provides the facilities for peer-to-peer communications between two programs. Programs and small computer systems use LU 6.2 to establish and control sessions with each other, without necessitating host mainframe participation.

\section{Magneto-inductive (MI) Head}

Type of read/write head technology used in 3380/3390 devices. Inductive $\mathrm{read} /$ write heads require a signal strength proportional to the velocity, which limits their optimal usage to large form-factor disks.

\section{Magneto-resistive (MR) Head}

Type of read/write head technology capable of reading magnetic bit streams independent of media velocity. These heads require fewer turns in the write coil, making them smaller and lighter.

\section{MB or Mbyte}

Megabyte-1 million bytes.

\section{MIPS}

Millions of Instructions Per Second; an approximate figure to denote a computer's raw processing power.

\section{OSF (Open Software Foundation)}

Non-profit organization decicated to delivering an open computing environment based on standards. Formed in 1988 to solicit technologies from industry and invites member participation to set technical direction.

\section{PB or Pbyte}

Petabyte, one million trillion bytes or 1,000 exabytes.

\section{PR/SM (Processor Resources/Systems Manager)}

IBM's logical partitioning facility, which allows the computer architecture to be separated from its physical implementation to provide a logical organization of resources that allows multiple operating systems to run on one physical CPU or complex.

\section{RAD (Redundant Array of Inexpensive Disks)}

A DASD technology that employs small (5.25-inch or smaller), low-cost disks. 


\section{Recovery}

The process of restoring a copy of the user's data from the backup inventory. Recovery may be used to replace an existing file or to create a new file with a new name.

\section{Retrieval}

A user-directed function that returns to the user an explicitly requested version of a file that had been archived, and optionally deletes it from the archive.

\section{Server}

A server provides functions for clients across an enterprise. A server could provide functions to other servers (such as a LAN server.)

\section{Shadowing}

Creating two concurrent copies of data allowing the system to recover dynamically.

\section{SLED (Single, Large, Expensive Disks)}

Disk technology employing form factors 8 inches or larger (used in IBM 3380, 3390).

\section{SMS (Systems Managed Storage)}

Term for the conceptualization of an architecture for attachment, management and reconfiguration of secondary storage. Among the SMS basic design goals is the separation of logical device management from physical device management.

\section{TB or Tbyte}

Terabyte, 1,000 GB or 1 trillion bytes.

\section{TLMS}

Tape Library Management System.

TMS

Tape Management System. 


\section{References}

1. WSRC-IM-91-18-1, SRS Computing Architecture.

2. WSRC-IM-91-18-2, SRS Computing Architecture Migration Guide

3. WSRC 1-01, Management Policies, MP 3.11, "Computing Standards and Guidelines."

4. WSRC 1-01, Management Policies, MP 3.12, "Computing Coordination and Integration."

5. WSRC-IM-92-23, SRS Computing Standards and Guidelines Reference.

6. Lawrence Berkeley Laboratory, ITR Assessment (draft), 12/13/91.

7. Katz, R H., G. A. Gibson, D. A. Patterson, "Disk System Architectures for High Performance Computing," Proceedings of IEEE, Vol. 77, No. 12, (December 1989).

8. Gartner Eighth Annual Storage Conference, April 22- 24, 1992. 


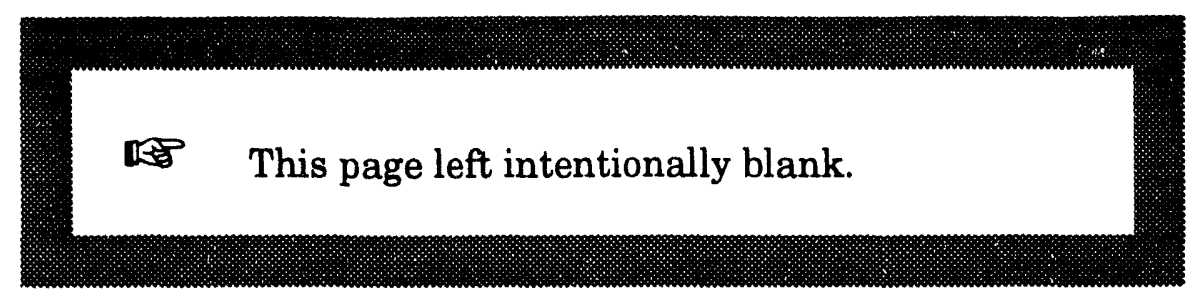



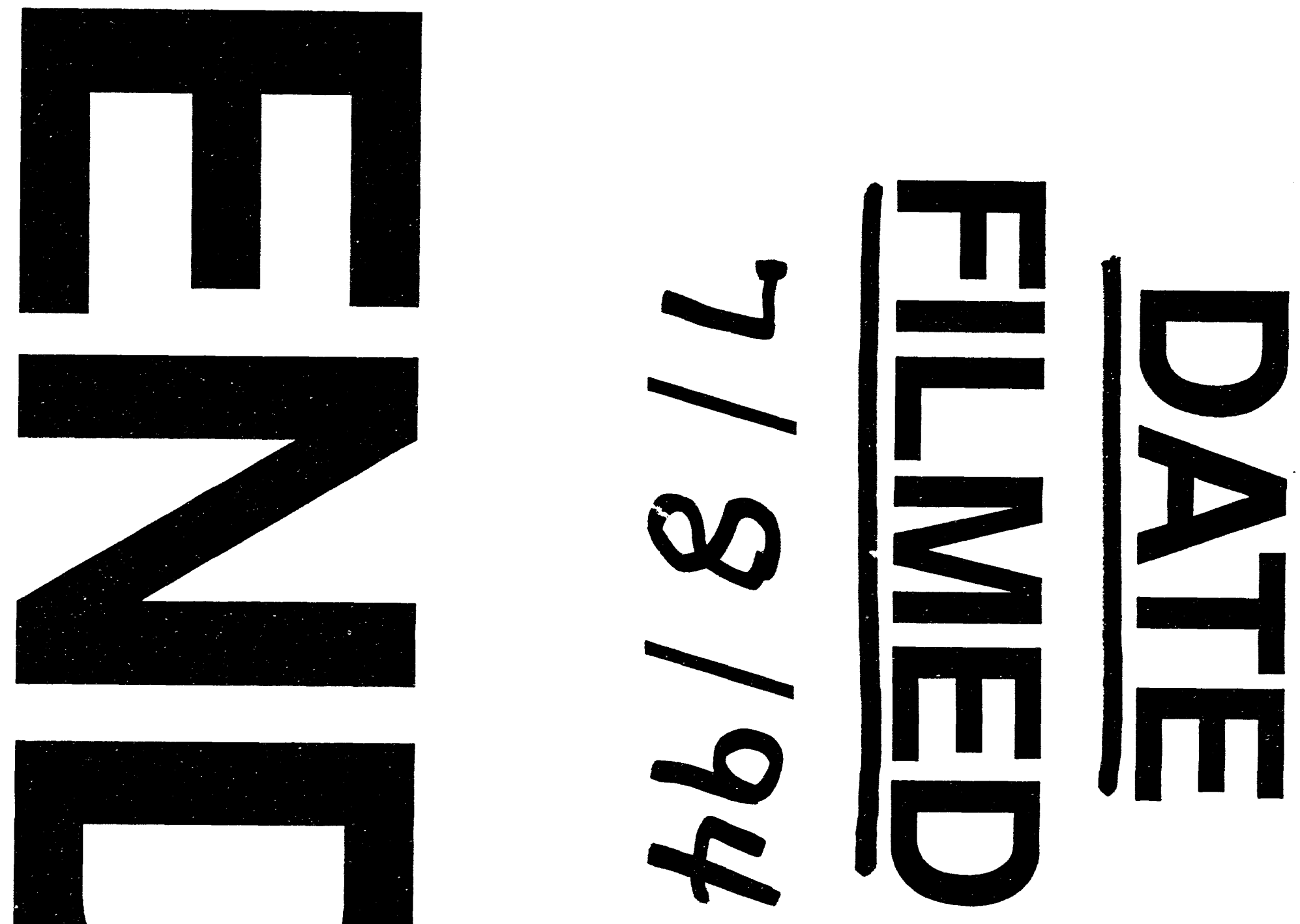
